\title{
La figure, un outil critique
}

Penser, raconter et comprendre le sens d'un territoire

The Figure, a Critical Tool. Thinking, understanding and sharing the meaning of a territory

\section{Andreea Grigorovschi}

\section{OpenEdition \\ Journals}

Édition électronique

URL : http://journals.openedition.org/crau/397

DOI : $10.4000 /$ crau.397

ISSN : 2547-5746

Éditeur

Éditions du patrimoine

Édition imprimée

Date de publication : 1 décembre 2014

Pagination : 91-101

ISBN : 978-2-7577-0379-3

ISSN : 1296-4077

\section{Référence électronique}

Andreea Grigorovschi, «La figure, un outil critique », Les Cahiers de la recherche architecturale et urbaine [En ligne], 30/31 | 2014, mis en ligne le 14 septembre 2017, consulté le 20 avril 2019. URL : http:// journals.openedition.org/crau/397; DOI : 10.4000/crau.397 


\section{La figure, un outil critique pour penser, raconter et comprendre le sens d'un territoire}

Dans le contexte actuel de transitions et mutations multiples (environnementales, sociales, politiques, spatiales, etc.), le champ disciplinaire de l'architecture urbaine est au centre d'un processus réflexif et autocritique, à la recherche des repères qui fassent sens. Cette recherche implique tout d'abord un repositionnement critique appelant à faire évoluer les approches, les méthodes et les concepts propres à ce champ disciplinaire. Au centre de cette redéfinition, le projet de territoire - entendu à la fois comme vision d'avenir et en même temps comme transformation spatiale envisagée - n'est plus compris comme garant des réponses à des problèmes bien identifiés et délimités dans le temps et dans l'espace, mais comme objet de savoir et comme "producteur de connaissances ${ }^{1}$ ». Suivant cette vision, nous pensons que les nouvelles logiques et dynamiques qui émergent des territoires en projet sont à saisir et à croiser, afin de nourrir les réflexions et les débats, en vue de la construction d'outils conceptuels et opérationnels pour le projet d'urbanisme contemporain $^{2}$.

1. Paola Viganò, Les Territoires de l'urbanisme. Le projet comme producteur de connaissance, Genève, MétisPresses, 2012.

2. L'ensemble de questionnements, hypothèses et réflexions qui font l'objet de cet article est issu de notre thèse de doctorat intitulée L'Architecture urbaine en débats. Du projet urbain au dessein métropolitain: cultures de projet en interactions. Strasbourg métropole rhénane et son récit urbanistique, menée depuis 2010 dans le cadre du laboratoire Amup-Ensa de Strasbourg, sous la direction de Cristiana Mazzoni. La deuxième partie de l'article, en lien avec notre étude de cas (Strasbourg), est issue de notre contribution à la recherche Strasbourg: la démocratie locale pour construire un récit sur la métropole durable, Cristiana Mazzoni (dir.), Laboratoire Amup/ Ensas, appel d'offres «Plate-forme 
Dans ce sens, dans notre travail d'observation et d'analyse des dynamiques projectuelles de la métropole strasbourgeoise, nous avons croisé à plusieurs reprises des formes de représentation du réel appelées figures de territoire (ou figures de projet de territoire) qui permettent de transmettre, par une image associée à des mots, le sens profond de l'espace et du lien avec la société qui l'habite. Cela nous amène à formuler notre hypothèse de départ selon laquelle la figure traduit, à travers une vision synthétique, le sens ou l'« horizon de sens ${ }^{3}$ " d'un territoire. À partir de cette idée, nous nous interrogeons sur la capacité des figures, en tant qu'outils conceptuels et opérationnels, de requestionner les cultures de "plan-masse " qui régissent les pratiques actuelles du projet urbain. De quelle manière pourraient-elles réinventer le processus de projet? Dans quelle mesure et de quelle façon pourraient-elles contribuer à l'évolution du projet comme objet/producteur de connaissance? Quelles sont leurs forces et leurs faiblesses? De quelle manière sont-elles construites (logiques ascendantes ou descendantes, lien avec le récit, l'imaginaire)? À partir de l'ensemble de ces interrogations, se dessine la nécessité d'un travail d'articulation entre la littérature existante sur le sujet et les observations recueillies sur le terrain.

Notre article s'inscrit dans cet effort réflexif de compréhension et de construction, et s'organise par conséquent en deux parties. Tout d'abord, nous ferons une exploration théorique sur la consistance de la notion de figure à partir d'un détour par la sphère sémantique et lexicale de la notion, pour revenir ensuite au champ qui est le nôtre, celui de l'architecture urbaine.
Au prisme de ces considérations théoriques, nous allons analyser dans un deuxième temps deux figures de territoire, telles qu'elles émergent dans le contexte strasbourgeois. Nous nous attacherons à comprendre leurs logiques et à saisir leur potentiel dans la démarche de conception.

\section{La « figura » : hybridations, dialectiques, ambivalences}

L'histoire sémantique et étymologique de la figure, ou plutôt de la figura, se dévoile dans le livre homonyme d'Erich Auerbach, qui retrace I'origine et l'évolution de la notion depuis l'Antiquité ${ }^{4}$. De cette histoire passionnante semble se dégager une constante: le rapprochement, parfois l'interférence, voire la superposition de la figura avec la notion de forma - deux concepts dont les sens s'entremêlent en permanence, parfois se confondant, parfois s'inversant. Avec la même racine que fingere ("modeler»), figulus (" potier»), fictor (" modeleur ») ou effigie (portrait), la figure fait penser à l'acte de façonner, au geste de sculpter, au travail de modelage, enfin, à l'opération de former. II n'est donc pas étonnant que sa signification initiale soit celle de forme plastique qui renvoie à la matière et au corps. Néanmoins, par rapport à ce sens originel, la notion de figure évolue, elle est nuancée, elle dépasse le sens de forme plastique pour glisser vers le conceptuel à travers l'idée de forme perceptible.

À partir de ce glissement, la figure ne se réfère plus uniquement à la matière, mais aussi au langage parlé et écrit (figure grammaticale, rhétorique, mais aussi mathématique et logique) et à celui visuel (illustrer un d'observation des projets et stratégies urbaines » (Popsu2), MEDDE-PUCA-AIGP (avec Jean-Alain Héraud, Florence Rudolf, Valérie Lebois, Luna d'Emilio, Andreea Grigorovschi, François Nowakowski,

Amandine Amat), rapport final, juillet 2013.

3. Bernardo Secchi, Première leçon d'urbanisme, Marseille, Parenthèses, 2005.

4. Critique littéraire et philologue allemand du début du xxe siècle, Erich Auerbach consacre la première partie de son ouvrage Figura à l'étude philologique de la notion. À partir de textes latins de l'Antiquité romaine, l'auteur montre l'importance du phénomène de l'hellénisation de la culture romaine et ses influences sur le concept. Voir Erich Auerbach, Figura, Paris, Belin, 1993 [1éd. 1944].

5. L'ambivalence mouvance-fixation a été explicitée par Philippe Dubois dans sa relecture du texte d'Auerbach. Si d'une part la dimension dynamique, celle de la "mouvance ", avec ses " effets de sens fluctuant » confère à la figure l'idée de flexibilité, de variation infinie, multiforme (comme un jeu qui ouvre une multitude de possibles, qui la laisse évoluer librement), d'autre part son " essence inaltérable » intervient quand la figure devient modèle, quand elle se fige à travers les codes, quand elle perd sa mouvance se rendant visible. Du dispositif évolutif, tel la « danse des figurae » et jusqu'à l'essence par excellence - telle 
livre de figures), faisant, de manière générale, appel aux sens (figure musicale ou chorégraphique) et à la pensée (d'où l'idée de se figurer quelque chose, de se le représenter mentalement, de l'imaginer). Ce passage entre le monde concret et celui des idées sous-tend plusieurs tensions, qui conditionnent et soulignent à leur tour le caractère complexe, déroutant et ambigu de la notion: entre le caché et le dévoilé (l'explicite et le tacite), entre mouvance et fixation ${ }^{5}$, entre sensibilité (ce qui est saisi par les sens) et intelligibilité (ce qui est compris par la raison), entre imitation et invention, entre déjà là et expression d'un désir d'existence (de ce qui n'existe pas, ou pas encore).

À travers ces ambivalences multiples émergent la nature hybride et la capacité de la figure à lier les éléments de nature différente: matériels et conceptuels, dits ou non-dits, etc. Cela nous amène à dégager l'idée qui est à la base de notre hypothèse, celle que tous ces rapports dialectiques que la figure sous-tend lui permettent de se promener librement entre espaces, paroles, images et pensées. À partir de ce constat, lier la figure aux champs de l'architecture et de l'urbanisme prend tout son sens.

\section{La figure dans le champ de l'architecture urbaine et son lien profond avec le projet}

Dans ces horizons disciplinaires qui sont les nôtres, I'utilité de la notion de figure a déjà fait l'objet de plusieurs explorations théoriques qui ont confirmé sa complexité polysémique. Dans l'effort de compréhension de la façon dont elle lie matérialités et idées de l'espace, la figure s'inscrit souvent dans une dimension représentationnelle: Pierre Pellegrino la rapproche des formes du langage ${ }^{6}$, alors que pour Frédéric Pousin elle appartient plutôt aux registres des instances figuratives ${ }^{7}$. Toutefois, certaines interprétations la situent dans une vision qui dépasse cette approche représentationnelle, lui conférant des acceptations hybrides liant «traces et discours" pour Bernardo Secchi ${ }^{8}$ ou encore se superposant à "toute chose structurée regardable » pour Michel Lussault?.

À une lecture attentive de ces auteurs, on peut émettre l'hypothèse que le statut et le rôle des figures d'espace se construisent à chaque fois en lien direct avec l'interprétation donnée au projet. À l'exception de Pousin, qui fait le choix de privilégier l'aspect graphique des figures, les autres auteurs interrogent les mécanismes de conception à partir de la question du sens, qu'ils explicitent de différentes manières. La portée des figures se définit pour chacun d'entre eux en fonction de son propre positionnement dans le champ de l'architecture urbaine et plus particulièrement au regard de la question projectuelle.

Pour Pierre Pellegrino ${ }^{10}$, qui privilégie I'approche sémiotique, la question du lien projet-figure, passe par la compréhension de l'architecture comme langage ${ }^{11}$. Analysée comme système de signes culturellement codés, l'architecture s'écrit à travers le projet vu comme un acte de conception codifié. Centrés plus particulièrement sur l'échelle de l'objet architectural, les efforts de l'auteur se dirigent vers la définition d'une grammaire capable d'établir les règles d'articulation entre apparence et consistance. Dans ce contexte, la figure émerge comme un élément de base du métalangage celle de l'« archétype »-, la figure manifeste cette relation dialectique de la «mouvance au sein d'une essence qui se maintient» (Auerbach). Voir P. Dubois, "La question des figures à travers les champs du savoir: Le savoir de la lexicologie: note sur Figura d'Erich Auerbach », in Dominique Château, François Aubral (dir.), Figure, figural, Paris, L'Harmattan, 1999.

6. Pierre Pellegrino, Figures architecturales. Formes urbaines, Genève, Economica,
Anthropos. 1994; P. Pellegrino, Le Sens de I'espace, livre III: Les grammaires et les figures de l'étendue, Paris, Economica, Anthropos, 2003

7. Frédéric Pousin (dir.), Figures de la ville et construction des savoirs: Architecture, urbanisme, géographie, Paris, CNRS, 2005; F. Pousin (dir.), "Pouvoir des figures », Les Cahiers de la recherche architecturale et urbaine, Paris, Éditions du patrimoine, 2001.

8. Secchi, op. cit. note 3 .
9. Michel Lussault, "L'espace avec les images », dans Bernard Debarbieux, Sylvie Lardon (dir.), Les Figures du projet territorial, La Tour d'Aigues, Éditions de I'Aube, 2003.

10. Pierre Pellegrino est architecte et docteur ès lettres et sciences humaines et membre fondateur et président d'honneur de l'Association internationale de sémiotique de l'espace.

11. Voir P. Pellegrino, op. cit. note 6; 
architectural traitant de la consistance, de manière complémentaire au type, qui lui, est intimement lié à l'apparence.

La figure, telle que l'auteur la comprend, n'est pas simplement un dessin, mais elle représente l'édifice projeté, à travers le travail de l'imagination. La figure produit ainsi du sens à partir de la systématisation de certains traits distinctifs de son expression et « montre la façon d'imaginer quelque chose qui n'est pas encore là, qui est en train d'être inventé ».

Le géographe Michel Lussault ${ }^{12}$, qui s'intéresse plus au projet à l'échelle territoriale, entend ce dernier comme processus et l'assimile à un « méta-récit multirationnel de l'action ${ }^{13}$ " fait d'énoncés sous des formes très variées: de la parole "spontanée », en passant par les textes et jusqu'aux icônes très construites. La figure devient alors un système langagier à part entière, qui n'est pas exclusivement discursif ni textuel et qui rend visible la relation individu-environnement. Contrairement à Pellegrino, chez Lussault la figure ne se réduit pas seulement à la question de la consistance, bien au contraire: très proche de l'image et de la représentation ${ }^{14}$, elle admet la composante visuelle (et par extension, celle physique) comme l'une de ses expressions privilégiées (sans pour autant nier l'importance du discours), allant jusqu'à inclure «toute chose structurée regardable ${ }^{15}$ ». Grâce à sa capacité de déployer une description qui intervient comme une « stase » dans le flux narratif de ce « méta-récit de l'action », la figure semble ainsi fondamentale pour le processus de projet.

Pour Bernardo Secchi ${ }^{16}$, qui considère que " ce qui fait la ville est l'organisation du discours », le projet d'espace, toute échelle confondue, est compris dans son rapport entre un savoir déjà là et un désir qui s'exprime. Autrement dit, pour l'urbaniste italien « il faut sortir de l'idée qu'un projet [...] peut être réalisé d'un bout à l'autre s'il ne construit pas un imaginaire collectif ${ }^{17}$ ". Ce qui se dégage de cette affirmation est l'ambition de l'auteur de comprendre, à travers le projet, le rapport entre l'espace et les pensées qui y sont liées. Les figures, telles qu'il les définit à partir de la rhétorique agissent à ces deux niveaux distincts : un niveau abstrait, quand les figures doivent être entendues comme «formes de pensée » et un niveau moins abstrait, où elles indiquent des «formes de la ville, de ses parties ou de ses architectures ». II repère ainsi, d'un côté les figures de discours - continuité, régularité, concentration et équilibre -, et de l'autre, les figures du regard - fragment, dispersion, hétérogénéité ${ }^{18}$. Investies par la force des imaginaires mobilisés à leur construction, les figures de Secchi dominent, s'opposent, agissent et alimentent à leur tour les imaginaires qui conditionnent le rapport de l'homme au monde.

Bien que ces interprétations n'aboutissent pas à une définition unanime de la figure d'espace, à la lecture croisée de ces auteurs, des hybridations se dessinent. Tout d'abord, ce qui ressort de ces trois regards est la capacité des figures à représenter. La référence à I'imaginaire (Secchi) et à l'imagination (Pellegrino) positionne la figure dans une condition intermédiaire entre représentation d'une réalité, comme «double analogique d'un objet » et en même temps le dépassement de cet état existant, en tant qu' « espace en plus » (Lussault). Autrement dit, la figure représente à la fois ce
P. Pellegrino, Emmanuelle P. Jeanneret, "Configuration, figure », Nouveaux Actes sémiotiques, n० 111, 2008. En ligne: epublications.unilim.fr/revues/as/, consulté le 27 janvier 2013.

12. Michel Lussault est géographe, professeur des universités et chercheur au laboratoire "Environnement, villes et sociétés » à Lyon. II s'intéresse à l'urbanisme et à l'aménagement de l'espace habité contemporain.
13. Ce « méta-récit multirationnel de l'action » suppose selon Lussault une multitude d'interactions et d'échanges entre les énoncés produits et diffusés par les différents acteurs qui prennent part au processus de projet. Voir M. Lussault, op. cit. note 9 .

14. Les textes de Lussault parlent tantôt de figure, tantôt d'image. Pour l'auteur, la figure-image est entendue au sens large: "Sans [...] la réduire au domaine de
I'iconique », son acceptation est étendue à celle de « représentation » comme "énonciation puissante d'une absence ", telle qu'employée en préalable par le philosophe Louis Marin (une référence pour Lussault). Voir M. Lussault, op. cit. note 9; M. Lussault, « Une problématique de l'image en géographie », in Christian Callenge, Michel Lussault, Bernard Pagand (dir.), Figures de l'urbain. Des villes, des banlieues et de leurs représentations, Tour, Maison des sciences de la ville, 1997; 
qui existe déjà et en même temps parle de ce qu'elle invente, expose ce qui n'existe pas sans elle et montre par conséquent un des ordonnancements possibles.

Un autre point qui semble communément accepté est la capacité des figures de lier et penser des éléments de nature différente " le texte (écriture architecturale) et le contexte (culturel, historique, social) » pour Pellegrino, " individus et environnement " pour Lussault, " objets, situations et événements qui ne sont pas toujours de même nature " pour Secchi. Lues à la lumière des remarques d'Auerbach, ces réflexions développées dans le champs de l'architecture urbaine confirment le fait que les figures d'espace/de projet fondent leur essence dans les tensions qu'elles sous-tendent et par rapport auxquelles elles doivent se positionner en permanence: entre caché et dévoilé, visible et invisible, mouvance et fixation, ou encore abstrait et concret.

Par ailleurs, le lien au discours (communément évoqué mais explicité de différentes manières), entendu comme forme d'expression des imaginaires, semble indiquer que la figure de territoire/de projet suppose une construction imaginaire complexe ayant la particularité de représenter une vision que l'on a d'un espace. Si l'imaginaire couvre l'ensemble des rapports, des sens entre les hommes et le monde, la figure semble traduire l'un de ces rapports, I'un des sens possibles d'un espace en particulier. Elle pourrait être définie comme le concentré d'une idée liée à un espace. Se dessine ainsi sa capacité de synthèse exceptionnelle, force et faiblesse en même temps. Si nous leur reconnaissons cette approche synthétique, les figures seraient peut-être en mesure de traiter la complexité des situations urbaines et territoriales contemporaines et pourraient par conséquent jouer le rôle de catalyseurs pour le processus de projet ${ }^{19}$.

\section{Strasbourg - de la ville mosaïque à la métropole des deux rives: figures de territoire en débat}

À Strasbourg, plusieurs figures émergentes portent les visions de ce territoire à la recherche de son sens. Leur consistance (objective/subjective, charge émotionnelle, symbolique, etc.) varie selon les conditions qui orientent les processus de leur élaboration. À partir des éléments théoriques relevés, comprendre la manière dont elles envisagent l'espace permet d'interroger non seulement leur rôle mais aussi l'ensemble du processus projectuel contemporain.

Depuis une dizaine d'années, Strasbourg est marquée par la volonté de passage de l'agglomération à la métropole. La tension entre ville de province et capitale européenne est une question qui anime le débat strasbourgeois actuel quand il s'agit de gouverner et de penser la ville. Les deux figures que nous regarderons de près traduisent justement cette ambiguïté: I'une - résultat de dynamiques ascendantes -, issue de la pratique des lieux et d'un imaginaire collectif appartenant à ceux qui fréquentent la ville; l'autre - plutôt portée par des logiques descendantes -, soutenue institutionnellement et issue d'une vision transfrontalière et d'une ambition métropolitaine affirmées ${ }^{20}$. Les récits qui les accompagnent sont issus d'une part des ateliers de travail et de discussion organisés avec les habitants ${ }^{21}$ et d'autre part, des discours portés par les acteurs institutionnels ${ }^{22}$.
M. Lussault, « La politique territoriale (trans) figurée ", EspacesTemps, n 62-63, 1996, p. 92-103; M. Lussault, "L'urbain sans figure », dans M. Lussault, Jacques Lévy (dir.), Dictionnaire de la géographie et de l'espace des sociétés, en ligne: remue.net/revue/TXT0407_MLussault.html, consulté le 2 janvier 2014.

15. Il est intéressant de remarquer comment, à travers cette acceptation, Lussault opère un élargissement de la définition de figure, incluant aussi la concrétisation du projet comme une de ses formes d'expression.

16. Bernardo Secchi est un économiste et urbaniste italien, professeur émérite de I'université de Venise (IUAV). Sa double posture, de théoricien et de praticien, et ses filiations aux courants de pensées italiens du xxe siècle, marquent la particularité de son regard sur les problématiques architecturales et urbaines contemporaines.
17. B. Secchi, Villes sans objet: La forme de la ville contemporaine, Centre d'étude conférences Mellon, 4 septembre 2008, en ligne: www.cca.qc.ca/system/items/1949/ original/Mellon15-BS.pdf?1241161735, consulté le 1er février 2014.

18. Voir B. Secchi, 2005, op. cit. note 3.

19. Pour comprendre plus en profondeur la force synthétique des figures, nous renvoyons vers les attributs que leur confère 


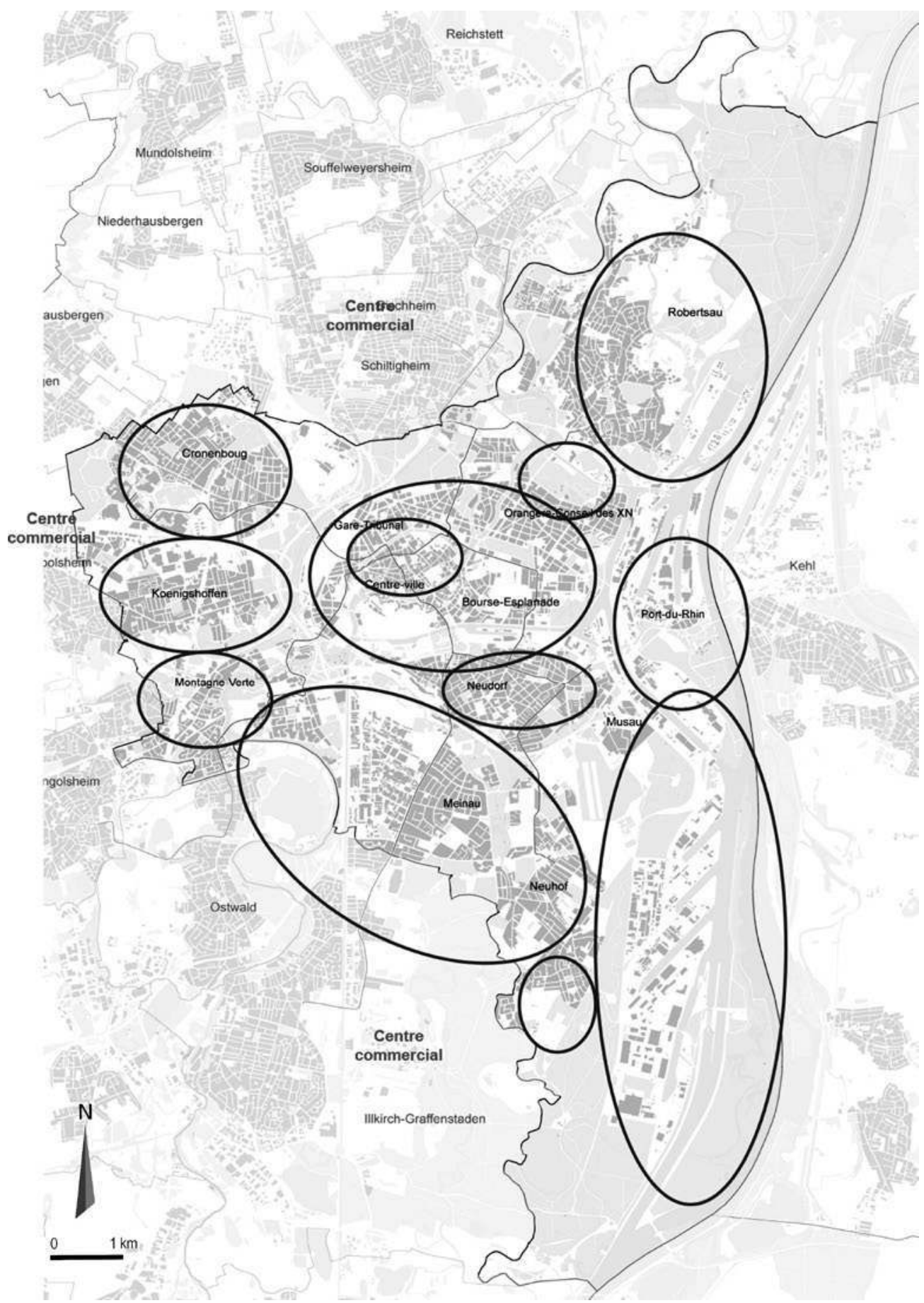

La figure de la ville mosaïque Source : Rapport de l'étude qualitative La silhouette urbaine de Strasbourg, Présentation

Powerpoint, IPSOS, 2011. 


\section{La figure de la ville mosaïque}

Issue d'un imaginaire habitant lié à ceux qui la pensent en la vivant, la figure de la ville mosaïque ${ }^{23}$, traduit la vision d'une "ville un peu morcelée, avec coupures», avec "une variété de style, un style par quartier». Des quartiers aux identités multiples et juxtaposées qui, ensemble, forment la ville. La ville mosaïque est l'expression de "l'unité dans la diversité », sans qu'elle cache pour autant ses lacunes, ses manques, ses facettes multiples et pas toujours réussies.

La force synthétique de la ville mosaïque réside en sa capacité à traduire non seulement la dimension visible de l'espace - celle des réalités matérielles -, mais aussi celle invisible, injectée par les lectures sensibles qui font appel à la pratique des lieux, aux affects, aux perceptions et aux souvenirs. La ville mosaïque est ainsi sillonnée par des "coupures", plus ou moins importantes, voire des "fractures », qui la rendent parfois difficilement praticable et inégalitaire. L'autoroute, le Rhin, la coupure centre/périphérie sont les indices physiques de frontières ressenties aussi socialement. Même si cette fragmentation spatiale rappelle également la figure de l'archipel, contrairement aux "îles», les pièces de la mosaïque ont des noms et des identités bien à elles: le quartier allemand, la presqu'île Malraux, la cité-jardin du Stockfeld, etc. Tantôt éloignées, tantôt juxtaposées et parfois même superposées, elles se distinguent et se reconnaissent les unes les autres.

Ces «quartiers avec des styles différents » ne sont jamais équivalents: I'importance du centre, par exemple, qui engendre "polarisation et hiérarchisation" est soulignée à plusieurs reprises: le centre est perçu comme la pièce privilégiée de la mosaïque - « centre pour les riches" associé au "carré d'or-quartier bourgeois». En effet, la "ville éclatée en de multiples zones", telle qu'elle ressort de l'enquête Ipsos en $2011^{24}$, voit sa pièce centrale s'imposer hiérarchiquement par la «puissance symbolique de la cathédrale », la faisant ressembler à « un village en grand » construit autour de son clocher.

Les habitants reconnaissent les évolutions, les transformations et les améliorations de leur ville mosaïque. Elle a notamment une particularité remarquable - le tramway qui permet la connexion de ces pièces et, en passant, arrive à gommer les erreurs. Le projet du tramway des années 1990, est vu comme « une aventure urbaine collective », comme un «fil conducteur » qui a su fédérer l'imaginaire collectif de la ville.

Si les réflexions théoriques positionnent la figure de territoire entre de l'espace en propre et de l'espace en plus, la figure de la ville mosaïque semble plutôt attachée à saisir une réalité qui est déjà là. Ainsi, cette figure est plus tournée vers l'interprétation plutôt que vers l'invention. Sans vouloir imaginer un état qui n'existe pas encore, elle s'intéresse aux liens entre les individus et leur cadre de vie, à travers une lecture qui privilégie l'approche sensible et subjective, parfois même idéalisée.

Ce regard est à comprendre, comme le suggèrent les auteurs consultés, dans les rapports qui s'établissent entre figures et récits. Nourrie par l'imaginaire collectif, la ville mosaïque se construit à partir de cet entrelacement de récits multiples qui la racontent au prisme de leur vécu. Mais, même si «l'agglomération (strasbourgeoise) s'est faite avec le tram ${ }^{25}$, force est de consta-
Lussault quand il les considère comme stases dans le flux narratif, capables de transmettre tout et en même temps. En revanche, le risque de glisser d'une vision synthétique à une vision simplificatrice n'est pas des moindres.

20. Les deux figures que nous présentons ici, celle de la "ville mosaïque » et celle de la « métropole des deux rives», ont émergé pendant l'atelier de projet «Silhouette urbaine » (dispositif de participation citoyenne, organisé par la CUS Communauté urbaine de Strasbourg (CUS) entre février 2012 et mars 2013) que nous avons observé (observation participante) et analysé dans le cadre du programme de recherche Popsu2. Si la première compte parmi les productions proprement dites de l'atelier, la deuxièmeseconde, faisant l'objet d'une présentation, rentre plutôt dans une phase d'apport de connaissances. Cette partie reprend et complète notre contribution dans ce sens, telle qu'elle a été rédigée dans le rapport final de recherche. Voir A. Grigorovschi, "L'Atelier urbain de Strasbourg. Productions de l'atelier de projet "Silhouette urbaine »: idées, thématiques, valeurs, figures de ville», recherche Strasbourg: la démocratie locale pour construire un récit sur la métropole durable.

21. Le mot « habitants " suggère le fait que ces ateliers s'adressent surtout au à un public large, ce qui n'exclut pas la présence des techniciens de la Ville ou encore des 
La figure de la métropole des deux rives

Source : Schéma de développement - projet EcoCités, "Démarche EcoCités. Strasbourg-Kehl, métropole des deux rives (Synthèse) ", publication CUS, avril 2009

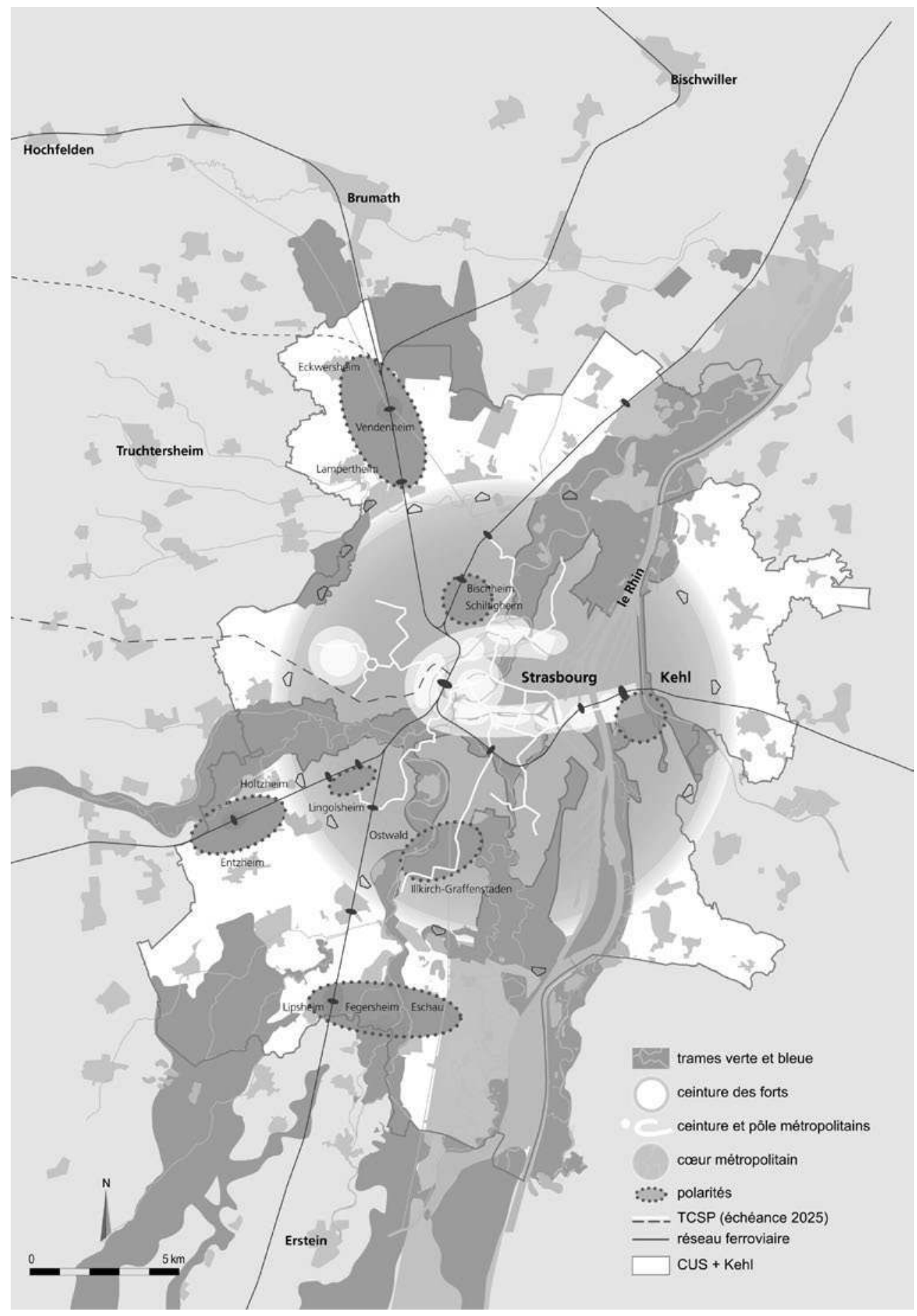


ter que la ville mosaïque, expression d'un imaginaire citadin circonscrit (des habitants de la ville de Strasbourg), se pense encore dans les limites administratives de la Ville. Plus encore, elle se confond parfois à l'idée d'une "ville village», avec son "harmonie bien particulière pour une ville -métropole " (lpsos), vivant d'abord dans la tête de ceux qui y aspirent et qui y voient un lieu idéalisé, alternative à un monde qui prend de l'avant. Malgré son indiscutable pouvoir de séduction, cet imaginaire - éloigné des réalités et du vécu hors du périmètre administratif de la Ville - semble déjoué par les réalités de l'urbain contemporain, de ce Strasbourg périphérique que les citadins mobilisés (habitants de la ville de Strasbourg) tendent à oublier et qui est, lui, marqué par des dynamiques et modes de vie qui couvrent un territoire bien plus large.

\section{La figure de la « métropole des deux rives "}

La métropole des deux rives émerge de la démarche "Écocités », portée par la Communauté urbaine de Strasbourg (CUS) en partenariat avec la Ville de Kehl en Allemagne ${ }^{26}$. Cette figure, porte-parole de deux revendications strasbourgeoises - la question métropolitaine et celle transfrontalière - donne à voir dans son expression graphique et dans sa traduction spatiale, une réflexion à l'échelle d'une agglomération élargie, correspondant aux périmètres de la CUS et de la Ville de Kehl. Sa structure est pensée à partir de trois trames structurantes - verte, bleue et transports en commun et à travers trois axes de développement - «le cœur métropolitain ouvert sur le Rhin ", " l'intensité urbaine » faite de multipolarités et mixités, et « la nature en ville ».
Si la ville mosaïque exprime le couple visible/invisible à travers les liens matérialités/vécu, la métropole des deux rives privilégie la dimension physique de l'espace (celle visible), tandis que la dimension invisible ne renvoie pas aux pratiques et aux sens, mais aux idées liées à une saisie plus objective du territoire. La dimension transfrontalière par exemple, hormis sa composante végétale et aquatique, s'appuie sur le croissant de la «ceinture métropolitaine " ${ }^{27}$. Imaginée comme la colonne vertébrale de la métropole naissante, cette dernière s'adosse et englobe les éléments considérés comme "faisant métropole », à savoir les institutions européennes, le « cœur métropolitain » correspondant au centre de Strasbourg, le centre de la petite ville allemande de Kehl et des grands équipements d'agglomération (Ciné Ccité, palais de la Musique et de la Danse, archives municipales et départementales, etc.) construits dans cette zone depuis les années 2000. Après le secteur des "Fronts du Neudorf » (grands équipements de l'agglomération et opérations immobilières au sud du centre-ville), l'attention se dirige vers l'est avec le projet « Deux-Rives », pensé autour du prolongement du tramway vers Kehl. La figure de la métropole des deux rives pose également la question du passage de la ville à la métropole. Sa traduction graphique suggère que cela se réaliserait à travers une continuité physique et une concentration d'équipements organisée le long d'une linéarité qui se veut transfrontalière.

Ainsi, entre sensible et intelligible, la représentation du territoire strasbourgeois, telle qu'elle émerge de cette figure, semble privilégier la deuxième seconde posture. Les pratiques et les attachements des individus experts (souvent) déguisés. De plus, les "Strasbourgeois » souvent évoqués dans les documents de présentation de ces dispositifs de concertation désignent exclusivement les habitants de la ville de Strasbourg.

22. Ces discours sont essentiellement exprimés par la voix des techniciens et professionnels de l'urbanisme qui travaillent pour ou avec les acteurs institutionnels.
23. L'ensemble des citations qui suivent et qui précisent cette figure - sauf indications contraires - correspondent aux propos tenus par les citoyens participants à I'Atelier de projet «Silhouette urbaine » de Strasbourg (février 2012-mars 2013).

24. L'enquête Ipsos effectuée à Strasbourg en janvier 2011, est consiste en une étude qualitative dont l'objectif est de faire ressortir la perception et la compréhension de la ville à partir du thème "Silhouette urbaine ». Cette enquête fait partie de la phase initiale du premier cycle de l'Atelier urbain de Strasbourg. Voir A. Grigorovschi, op. cit. note 20.

25. Propos de René Tabouret (ingénieur retraité, ancien conseiller municipal et un des experts de l'Atelier urbain de Strasbourg), recueillis lors de la dernière séance de l'Atelier de projet « Silhouette urbaine », le 22 janvier 2013. 
conditionnent moins explicitement cette vision qui s'appuie essentiellement sur des éléments physiques et des développements plus liés à la raison qu'aux perceptions et affects. De plus, si la figure de la ville mosaïque renvoie plutôt à l'espace en propre, celle de la métropole des deux rives semble privilégier le dépassement d'un état existant. Plus tournée vers l'imagination et l'invention (issue d'une démarche de proposition propre aux "spécialistes » ${ }^{28}$ ), elle exprime un désir, voire une ambition soutenue par la municipalité. Pas nécessairement contradictoire aux désirs et volontés de l'imaginaire collectif strasbourgeois, elle parle néanmoins peu des spécificités, des pratiques et du sens des territoires déjà là, se limitant également aux périmètres administratifs. On peut s'interroger ainsi sur la pertinence de l'échelle mise à l'étude, sur la place du Rhin au centre des deux rives ou encore sur les spécificités et les pratiques territoriales métropolitaines qu'elle évoque.

Construites ainsi, les deux figures strasbourgeoises, bien qu'elles ne s'opposent pas, ne communiquent pas entre elles pour autant. Elles sont toutes deux issues d'une recherche de sens par rapport à un contexte local. Elles témoignent aussi d'une condition physique, politique et sociétale particulière qui s'exprime différemment à travers les imaginaires mobilisés, leurs pratiques et leurs désirs. Si la première $s^{\prime}$ inscrit dans le temps de l'expérience idéalisant même parfois une réalité qui n'existe plus, la deuxième seconde est tournée vers l'avenir et portée par l'ambition métropolitaine et transfrontalière ${ }^{29}$. De plus, la ville mosaïque est essentiellement le fruit d'une approche subjective et sensible, alors que la métropole des deux rives relève d'une démarche qui privilégie un raisonnement plus objectif.

Cependant, comme le suggèrent Secchi et Lussault, les figures, permettraient de dépasser une représentation issue exclusivement de la pure imagination du seul concepteur, pour intégrer des éléments d'un imaginaire collectif déjà là, lié à un lieu et à son histoire particulière. Inversement, les figures peuvent dépasser la simple représentation d'un état existant ou une vision basée fondée exclusivement sur un attachement personnel et subjectif pour s'ouvrir vers des interprétations et des déclinaisons multiples, à travers le travail de l'imagination. Autrement dit, le sens du territoire que la figure transmet peut s'enrichir et se bâtir en lien avec d'autres figures qui subsistent dans les imaginaires individuels ou collectifs mobilisés.

Cela nous amène au lien qu'elles établissent avec la démarche de conception et leur place dans le processus de projet. Dans une posture où le projet d'espace est entendu comme producteur de connaissance plus que comme solution à un problème donné et localisé, trouver les figures de territoire qui subsistent dans l'imaginaire collectif nous apparaît comme la première étape du processus projectuel. Ces figures pourraient alimenter ou évoluer vers des figures "de projet", qui réinterrogeraient ensuite le sens des premières. Le passage des sens profonds synthétisés dans les figures serait ainsi à la base de leur rôle de "catalyseurs " du processus, permettant de structurer le projet autour d'idées phares, porteuses de sens. De par cette tension qui les fait osciller entre mouvance et fixation, les figures
26. Les propos et les locutions apparaissant entre guillemets dans la partie qui suit sont issus, d'une part, de la présentation de la démarche "Écocités » faite à la septième séance de l'Atelier de projet «Silhouette urbaine » (23 octobre 2012) par Stéphanie Strasser (technicienne, direction de I"Urbanisme de la CUS), et, d'autre part, du texte de la plaquette de présentation. Voir Démarche ÉcoCités, Strasbourg-Kehl, métropole des deux-rives (synthèse), publication CUS, avril 2009.
27. Cette dernière se superpose majoritairement à l'ancienne ceinture des glacis, classée comme zone non aedificandi (non constructible) de 1922 jusqu'aux années 1990, quand cette loi a été abrogée, ouvrant la voie à l'urbanisation.

28. Nous faisons ici référence aux " experts », professionnels de l'urbanisme, de l'architecture et de l'aménagement, maîtres d'œuvre ou techniciens de la Communauté urbaine.
29. Nous rappelons ici les deux catégories établies par Reinhart Koselleck, celle de l' " espace d'expérience » et celle $d^{\prime}$ " horizon d'attente ». Voir R. Koselleck, Le Futur passé: contribution à la sémantique des temps historiques, Paris, École des hautes études en sciences sociales, 1990 [1éd. 1979]. 
strasbourgeoises pourraient, et nous soutenons cette hypothèse, interroger et lier connaissances et attentes, analyse et projet: injecter la vision sensible venant du terrain pour enrichir une lecture intelligible, saisir l'imaginaire du déjà -là pour inventer son évolution vers un autre état possible, comprendre la ville mosaïque (ou d'autres figures de territoire à l'œuvre) pour imaginer la métropole des deux rives.

\section{Conclusion sous forme $d$ 'hypothèses}

Nous avons essayé d'explorer dans ce texte la portée théorique de la figure et deux de ses expressions telles qu'elles émergent dans le contexte particulier de Strasbourg. Cette exploration nous permet de dégager, sous forme d'hypothèses, certaines forces et risques que les figures de territoire, comprises comme outils conceptuels et opérationnels pour le projet d'urbanisme contemporain, présentent. Tout d'abord, leur capacité d'intégrer des éléments de nature multiple (espaces, paroles, images, idées, perceptions) semble un atout majeur pour saisir la complexité grandissante des territoires contemporains. Par ailleurs, leur caractère souple leur permettant d'évoluer et de se décliner tout en gardant leur essence, semble leur donner la capacité de gérer l'incertitude et d'intégrer les imprévus. Leur processus de construction pourrait ainsi correspondre à l'ouverture d'un espace de rencontre et de dialogue entre des points de vue pluriels. Néanmoins, tirer profit de ces atouts ne semble pas toujours facile quand il s'agit de la construction d'une figure de territoire en particulier. Cela est dû au fait que la capacité de synthèse propre aux figures ne se délivre pas à l'évidence aisément.
Elle semble conditionnée par une approche subtile, où enjeux et désirs se conjuguent nécessairement à une connaissance fine et sensible du terrain. Dans ce sens, l'une des questions qui restent ouvertes est celle des méthodes à inventer pour trouver l'équilibre entre les différentes sphères - matérialités, intellects et sensibilités - afin d'éviter de tomber dans des représentations simplistes ou partielles. Une autre force des figures réside en leur qualité de catalyseurs qui pourrait assurer les liens entre approche intelligible et sensible de l'espace, ou encore entre analyse et projet. Conjuguée avec leur capacité critique, cette condition d'intermédiaire semble les placer comme outils conceptuels et opérationnels intéressants, capables de penser la forme des territoires en lien avec leurs "horizons de sens". Autrement dit, au moment où la démarche de projet a plus que jamais besoin d'outils réflexifs, la figure permettrait d'interroger non seulement les configurations spatiales, mais aussi l'adéquation entre les concepts mobilisés et les échelles de réflexion questionnées. Quels seraient alors les rapports entre figures et étendues spatiales et temporelles? Cela reste une autre question encore ouverte. Enfin, compte tenu de toutes ces considérations, la figure pourrait devenir sinon une notion fondatrice, du moins un outil conceptuel qui saurait renforcer la recherche en architecture urbaine et penser l'articulation entre la théorie et la réalité spatiale, en vue d'un positionnement à trouver face aux mutations plurielles de l'époque contemporaine. 